\title{
A Novel Architecture for Searching Items in Wireless Personal Area Networks (WPAN) with Zigbee Technology
}

\author{
R. Priya \\ Ph.D Part-Time Scholar \\ Department of Computer Science \\ Bharathiar University \\ Coimbatore - 641046
}

\author{
G.P. Rameshkumar \\ Professor \& Head, \\ Department of Computer Science, \\ SNR Sons College (Autonomous), Coimbatore-6
}

\begin{abstract}
There are many technologies that are already available for searching any items with mobile phones. But in this method, user should have mobile phone in working condition. This research paper has been developed to search the frequently used homely products during emergency. If any user wants to search items, this application is very much useful.
\end{abstract}

\section{Keywords}

Wireless network, zigbee technology, sensor, Bluetooth

\section{INTRODUCTION}

Now-a-days, people's day to day life has become more faster which creates stress and tension. If user want to go out urgently, at that time they may miss some important things such as house key, car key or bike key (etc..). At that moment user's mind will be completely blank and user has to spend their valuable time in searching those items. To avoid this problem, the proposed model has been developed for searching the items within a particular area like Wireless Personal Area Network.

\subsection{Wireless Personal Area Network (WPAN)}

The Wireless Local Area Networks has obtained a great success in dealing with wireless networks in the particular area. In recent trends, the capabilities of WLAN is increased to a greater extend and also many new methods have been developed for the growth of WLAN. In addition, there are also many standard protocols are available for wireless connectivity. Wireless Personal Area Network (WPAN) has been introduced after WLAN of low cost [1].

WPAN is a low range network. It serves to interconnect all the ordinary computing and communicating devices which covers a few dozen meters [2]. It is mainly used for connecting peripheral devices like printer, cellphone, computer, home appliances etc., without using wired connection. Several technologies are available for this network like bluetooth, homeRF, WiFi, zigbee, WiMAX etc.

\section{EXISTING METHOD}

Already users have some technology for searching items with Bluetooth technology in Mobile phone. These methods can be used in iPhone or Android phone only. For example, Tile, TrackR and Duet Reviewed and so on. But in these methods, user should have mobile phone in his hand. Also, the searching software should be installed in the mobile phone for searching items with Bluetooth. In these methods, each item has sensor chips and mobile phone can connect with sensor chips through Bluetooth. In this method, mobile phone should be in working condition. And the mobile should not be missed, because, this methods is executable only with mobile phone through blue-tooth.

Existing methods are mainly used for key-finder applications with blue tooth technology and working with battery. If user wants to find the car key, Vehicle key finder system is very much used to find the misplaced key. This system mainly saves the time and energy. If user missing the car key, user can be found the car key by single click a button from the Android phone through Bluetooth technology and also buzzer indication is given from key. So it is very easy method to find the car key within few seconds [3].

User should know how many days the battery charge lasts and how long sensor batteries would last before changing the battery. These wireless key finder applications are used in Public Park and home also. It would be tested in various places with various distances, alarm or alerts were audible in barrier places also. In this case, if two or more wireless sensors are working simultaneously in the same place, the Bluetooth connectivity would be disturbed with one of the sensor or others.

Tile, TrackR and Due Reviewed

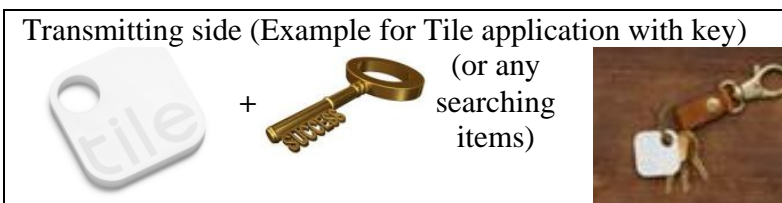

Receiving side

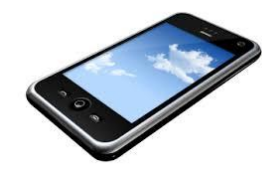

With sensor chips (Tile, TrackR and Due Reviewed) with Bluetooth technology

Fig 1. Existing method of searching items

If the user wants to search any items, the items should have sensor chips like Tile, TrackR and Due Reviewed application etc,. with it. 
Till now, the rechargeable batteries are used for Tile, TrackR and Due Reviewed applications. This was a challengeable due to the frequent recharging the batteries [4].

Tile is a square shaped tracker attached to the item to be tracked like bike key, home key, any bag or more. Now a days, Tile gives support very much for Android Smartphone to find missed or stolen items, by allowing larger group of people using their mobile phones. If user wants to search any items, first the user must activate wireless network within his mobile phone through Bluetooth, and then connect to the item to be searched based on sensor chips through searching application.

\subsection{Zigbee}

ZigBee is a wireless network and aimed at remote control and sensor applications which is suitable for any operation in

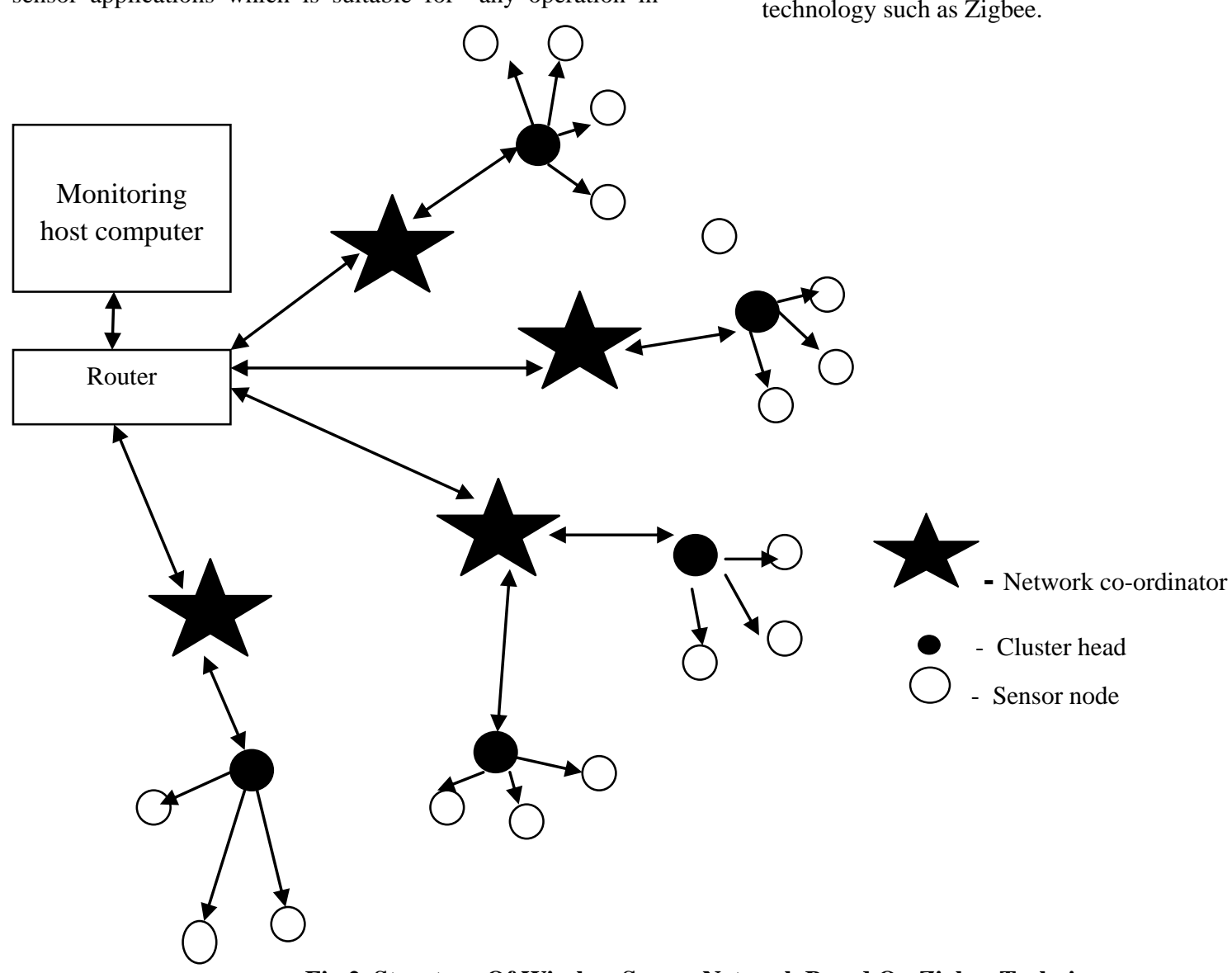

Fig 2. Structure Of Wireless Sensor Network Based On Zigbee Technique

\section{Characteristics of Zigbee}

- Very low cost

- Easy for implementation

- Reliable data transfer from one place to another place

- It is applicable only within a particular place (Short range operation)

- Very low power consumption

- Adequate security features

- Small size packet device

- It is based on wireless sensor network harsh radio environments and in isolated locations [5]. Zigbee is one of the wireless protocols used in Wireless Personal Area Networks. Already zigbee is used to detect the leaking gas or fire like Infrared Detector, Temperature Sensor, Smoke Detector and Gas Sensor with alarm message remotely. It is mainly used in security system to prevent fire or gas leakage in a particular building. Security is a very vital issue in home or other places. Zigbee technologies are very less power consumption security alarm system developed by WSN and GSM Technology. It consist of microcontroller and wireless sensor network, GSM, mobile phone etc. This hardware have sensor chip for receiving and sending the datas. Now a days, Security Monitoring Systems are very popular in office, home or any other buildings. Not only in home or any devices, it is also used for individualized office automation applications. This proposed model has been developed with wireless sensor technology such as Zigbee.

\subsection{Sensor node}

The sensor node is a basic unit of the wireless sensor network and it is used for data analog / digital conversion. The processing module of sensor node is responsible for controlling the whole operation and binary information transmitted from other devices. It is mainly used for exchanging information and controls the receiving or transmitting data [6].

\section{LITERATURE REVIEW}

Now-a-days, wireless sensor network works at different places such as fire detection system, leaking gas detection, open/close status of gates and doors in large warehouses, industrial automation, traffic monitoring, air traffic control, 
robot control, monitoring weather conditions, monitoring medical devices, viedo surveillance, automated smart homes etc. Some of the applications are given below:

- Forest fire detection system is one of the wireless sensor system and it consists monitoring nodes, base stations, communications systems, Internet access. Forest fire detection system has large number of the different type of sensors to monitor the value change including temperature, humidity, smoke or gas detector etc. All sensor nodes are divided to some cluster. Each cluster is equivalent to a relatively fixed self-organizing network. All the data is stored to the particular database [7].

- Industrialization increases air pollution by releasing the unwanted gases in environment. Sensor nodes used to detect percentage of pollution by using zigbee technique [8].

- The Air Traffic Control (ATC) tower is located in or near the aerodrome and usually has visual contact with aircraft. Tower may consist of a local controller, ground control and clearance delivery. The local controller is responsible for all traffic within the control zone and on the runways, the ground controller for all traffic on the taxiways. It may provide all kind of information and especially traffic information to the pilot [9].

- The Traffic density is increasing in developing countries, it needs to implement advance intelligent Traffic signals to replace the Conventional manual and time based Traffic signal system. These Advance intelligent traffic signals have central microcontroller at every junction which receives data from tiny wireless sensor nodes place on the Road. The sensor nodes have sensors that can detect the presence of vehicle and the transmitter wirelessly transmits the traffic density to the central programmable microcontroller. The Microcontroller makes use of the proposed programmed algorithm to find ways to manage and regulate traffic in a systematic manner efficiently [10].

\section{PROPOSED MODEL}

This proposed model is mainly focused on sensor application connected with the frequently used items to perform searching through buzzer mechanism. In this model, zigbee electronic device used for connecting sensor chip with the help of switches. In this block diagram, the proposed model have three section such as transmitting section, receiving section, and communication channel between the transmitting section and receiving section. The communication channel consists of Zigbee Transmitter, Zigbee Microcontroller and Zigbee Receiver. In transmitting section have electrical switches for Mobile phone, TV remote, Bike key, Car key etc. If user press the switch, the electrical switch connection with zigbee transmitter and Microcontroller. In receiving section have searching items with sensor chips. Zigbee receiver activate with Zigbee transmitter, and find out the items, and then alarm make noise for detecting items.

A wireless sensor network consists of multiple detection station called sensor nodes and each node is very small, lightweight and portable. Every sensor node consists transducer, microcontroller, transceiver (transistor and receiver) and power source. Transducer generates electrical signals, micro-controller is used for stores the sensor output, transceiver receives commands from a central computer and transmits data to the other parts and the power sources for each sensor node is derived from a battery as a source.

In this block diagram, the proposed model have three parts such as transmitting section, receiving section, in between the both section of wireless sensor network with micro-controller section. In transmitting section have electrical switches for Mobile phone, TV remote, Bike key, Car key etc. If user press any switch, the electrical switch connection with zigbee transmitter and Microcontroller. In receiving section have searching items with sensor chips. Zigbee receiver activates with Zigbee transmitter, and find out the items, and then alarm make noise for detecting items.In this method, first user should set electric switches for each item in his house like home switches.

\section{Wireless communication phases}

\section{$\underline{\text { Transmission Section }}$}

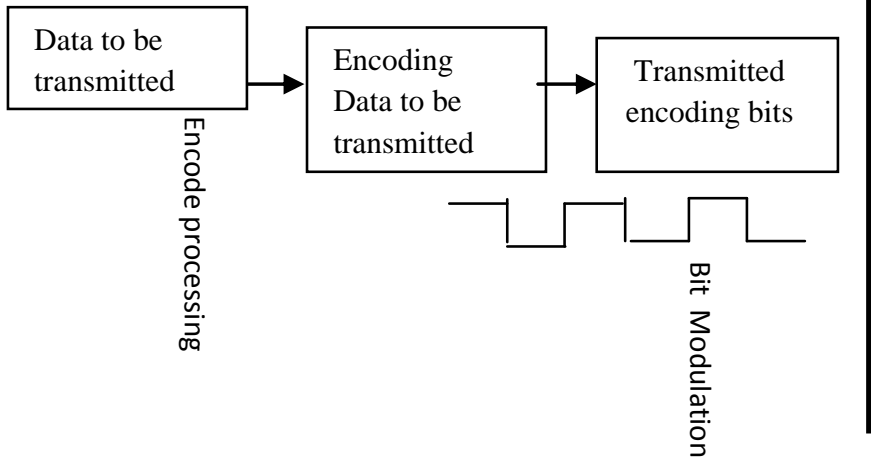

\section{$\underline{\text { Receiving Section }}$}

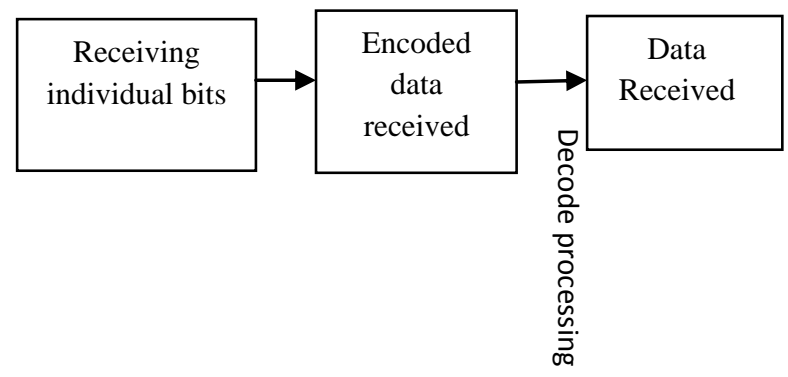

Fig 3. Phases of wireless communication 


\subsection{Architecture of proposed model}

The architecture of proposed model consists of three sections as Transmitting section, Receiving section and communication channel. The transmitting section consist of individual switches for the items such as mobile phone, TV remote, Bike key and Car key. The Receiving section consists of respective alarms for the same. Communication channel consists of four transmitters for the corresponding switches. For example, the switch of the mobile phone is connected to its respective transmitter T1. Similarly, other items are connected to its respective Transmitter T2, T3 and T4. The output lines from all the transmitters T1 to T4 are connected to a single microcontroller. Microcontroller is used to control the incoming and outgoing signals between the transmitters and the receivers. Microcontroller gets connected to the voice and playback circuit with amplifier, which is responsible for controlling the quality of signals. Next to the voice and playback circuit with amplifier, there are four receivers R1, $\mathrm{R} 2, \mathrm{R} 3$ and R4. Each receiver receives the incoming signal from the transmitter and send it to the respective alarm which is on receiving section. Then the receivers $\mathrm{R} 1, \mathrm{R} 2, \mathrm{R} 3$ and $\mathrm{R} 4$ are connected to its alarm.

\section{Communication channel}

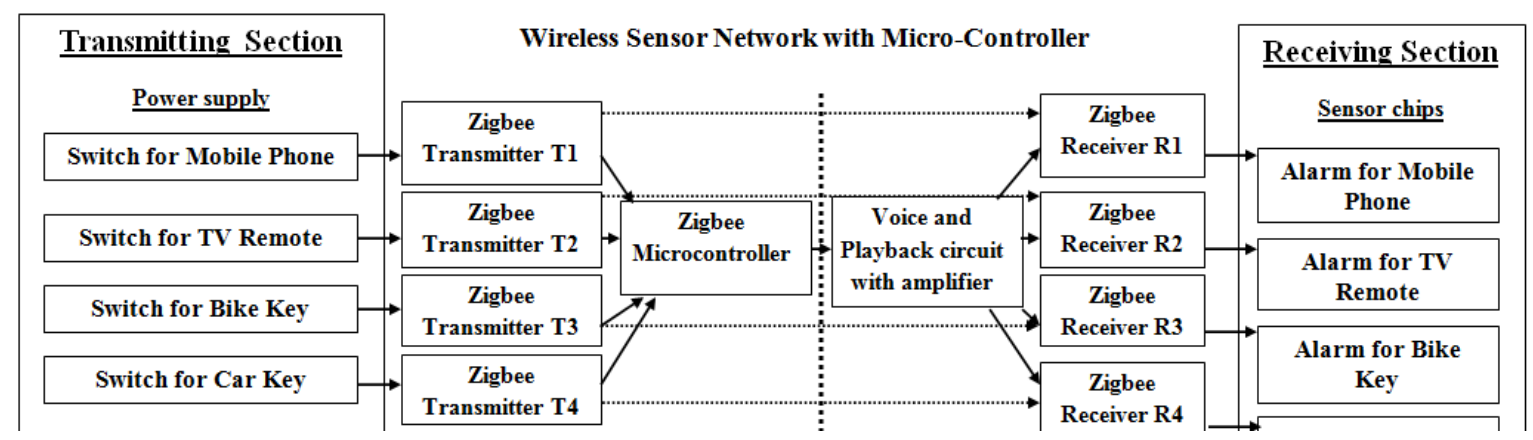

Fig 4. Block diagram for proposed model

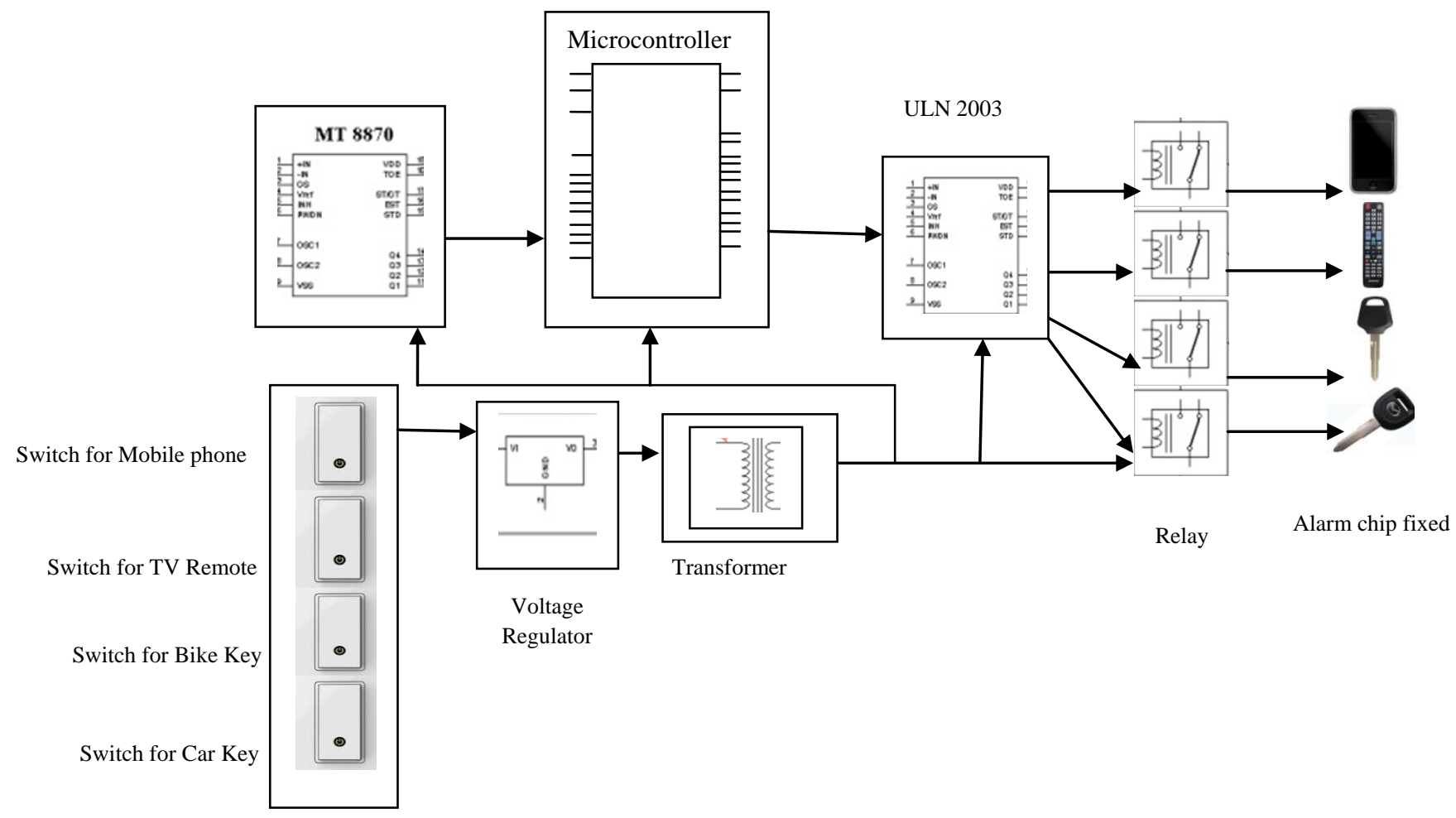

Fig 5. Basic circuit design for proposed model

In fig 5 , when the switch is pressed for finding an item, the signal from the switch is send to the transformer through the voltage regulator and is converted to binary digits with the help of decoder. The binary digits are sending to ULN2003 circuit. ULN2003 circuit is used in low voltage to switch the respective alarm through relay driver.

\subsection{Advantages of the proposed model}

- Time consuming: The main advantage of the proposed model is time consuming. However, it is easy to identify the frequently used accessories like mobile phone, TV remote and so on. Whenever, the user's in need of those accessories, the users time is consumed just by pressing 
the respective switch of that accessory by this, alarm will indicate where the device is located.

- $\quad$ Reducing stress and tension: The people in this century are so busy. Hence by using this proposed model, they can save their time to a greater extend.

- Easy to handle: Even small children could handle the device.

\subsection{Disadvantage of the proposed model}

- Cost: The proposed model requires an individual transmitter and receiver for every accessories which are included in it. This increases the amount to be spent for transmitters and receivers.

Comparison of Existing method and proposed model

\section{FURTHER ENHANCEMENT}

To overcome the only disadvantage of the proposed models the cost involved in transmitters and receivers has to be reduced. Instead of using the individual transmitters and receivers for each items, a single transmitter and receiver is to be used as common for all the items. The block diagram explains the further enhancement model clearly.

The architecture of enhancement model consists of three sections as Transmitting section, Receiving section and communication channel. The transmitting section consist of individual switches for each items. The Receiving section consists of respective alarms for the items. Communication channel consists of single transmitter. The output lines from the transmitter will be connected to a single microcontroller. Microcontroller is used to control the incoming and outgoing signals between the transmitter and the receiver. Microcontroller gets connected to the voice and playback circuit with amplifier, which is responsible for controlling the quality of signals. The voice and playback circuit with amplifier is connected to single receiver; it receives the incoming signal from the transmitter and send it to the respective alarm which is on receiving section.

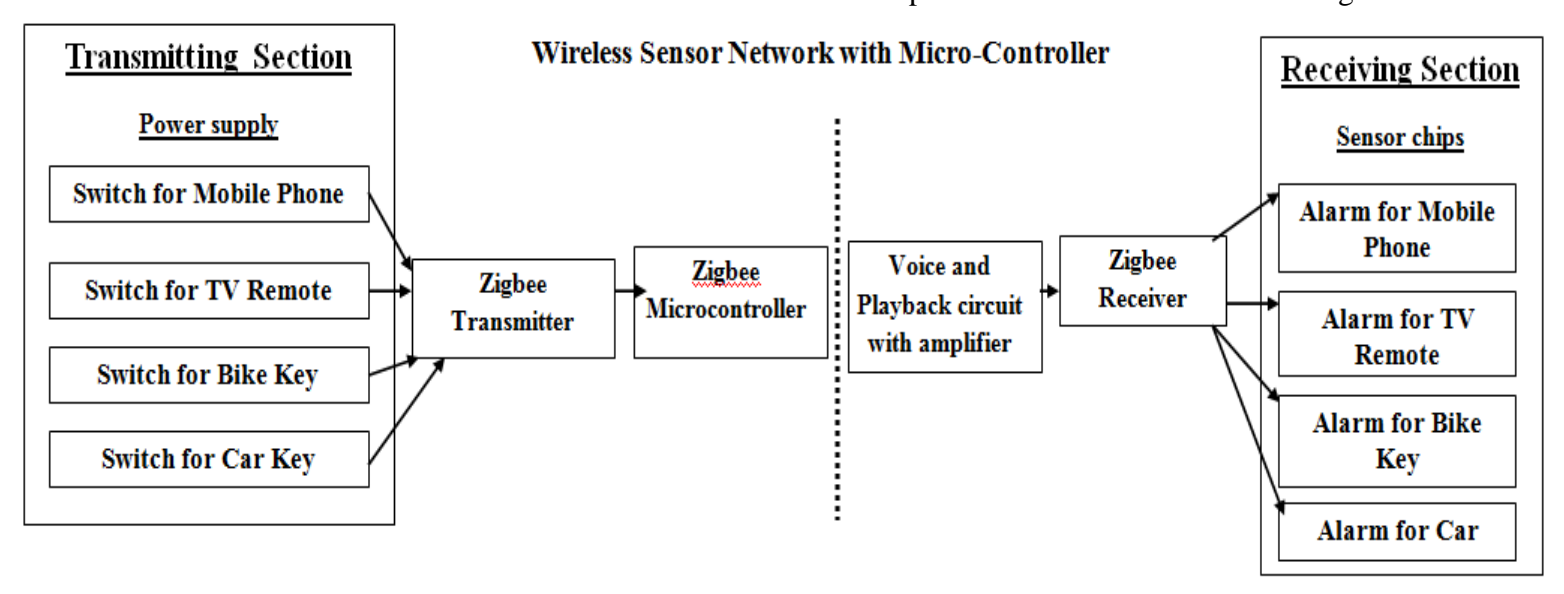

Fig 6. Block diagram for further enhancement

\section{DISCUSSION ABOUT PROPOSED MODEL}

- It is possible to identify the location of mobile phone by calling from other mobile phone. To do so, the mobile phone which the user is searching for must be in ON mode and not in silent mode. But in this proposed model, it is not necessary that the mobile phone must be in ON condition. And also this method is very useful while the mobile phones are in silent mode also.

\section{CONCLUSION}

This method mainly pointed out for unique advantages such as safety data transmission, flexibility in building the network and low cost. The topology structure of the system is an adaptation of a cluster-tree. Compared with a reticular structure, a cluster-tree structure can be built more easily and the information path takes less memory space. At the same time, the chain structure needs to be stable and its scale is limited, which needs to be improved in future investigations.

Peoples day to day life becomes more faster. Though user wants to save their time from spending it in searching the frequently used items. For example, if users want to go out urgently, at that time, house key, car key or bike key (etc..) may missing. At this time, users spend their time for searching such items. To avoid such problem, the proposed model has been used for searching the items.

\section{REFERENCES}

[1] Ivan Howitt and Jose A. Gutierrez, "IEEE 802.15.4 Low Rate Wireless Personal Area Net work Coexistence Issues",0-7803-7700-1/03 /\$17.00 (C) 2003 IEEE, page No.1481 to 1486

[2] http://ccm.net/contents/834-wpan-wireless-personalarea-network

[3] Sindhoori S, Dr. N. Sathish Kumar2, "Android based Secured Vehicle Key Finder System", International Journal Of Modern Engineering Research (IJMER), ISSN: 2249-6645, Vol. 5, Iss. 5, May 2015

[4] EDN Network, "Tintag Takes on Tile \& TrackR Bravo with the First Rechargeable Item Tracker", Tintag September 03, 2014

[5] http://www.radioelectronics.com/info/wireless/zigbee/zigbee.php 
[6] Junguo ZHANG, Wenbin LI, Ning HAN, Jiangming KAN, "Forest fire detection system based on a ZigBee wireless sensor Network", Research Article, Front. For. China 2008, 3(3): 369-374, DOI 10.1007/s11461-0080054-3

[7] Nandini Ammanagi, Yogesh Prajapati, Hardeep Purswani, Milesh Janyani and Atharva Nandurdikar, "Automation in Air Traffic Control using ZigBee and Ultrasonic Radar", International Journal of Emerging Technology and Advanced Engineering, Website: www.ijetae.com (ISSN 2250-2459, ISO 9001:2008 Certified Journal, Volume 5, Issue 4, April 2015)

[8] P.Vijnatha Raju, R.V.R.S.Aravind, B Sangeeth Kumar, "Pollution Monitoring System using Wireless Sensor Network in Visakhapatnam", International Journal of Engineering Trends and Technology (IJETT) -
Volume4Issue4- April 2013, ISSN: 2231-5381 http://www.ijettjournal.org Page 591

[9] P.S. Jadhav, V.U. Deshmukh , Vidya Pratishthans College of Engineering, Baramati, Pune University, "Forest Fire Monitoring System Based On ZIG-BEE Wireless Sensor Network", International Journal of Emerging Technology and Advanced Engineering, Website: www.ijetae.com (ISSN 2250-2459, ISO 9001:2008 Certified Journal, Volume 2, Issue 12, December 2012)

[10] Rashid Hussian, Sandhya Sharma,Vinita Sharma, Sandhya Sharma, "WSN Applications: Automated Intelligent Traffic Control System Using Sensors", International Journal of Soft Computing and Engineering (IJSCE), ISSN: 2231-2307, Volume-3, Issue-3, July 2013 